\title{
Adiabatic cycles in quantum systems with contact interactions
}

\author{
Atushi Tanaka, Taksu Cheon \\ Department of Physics, Tokyo Metropolitan University, Hachioji, Tokyo 192-0397, Japan \\ tanaka-atushi@tmu.ac.jp, taksu.cheon@kochi-tech.ac.jp
}

\begin{abstract}
An adiabatic cycle involving the $\delta$-function potential is shown to excite Bose particles confined in a one-dimensional box. We consider a cycle, during which a wall described by a $\delta$ potential is applied and its strength and position are slowly varied. When the system is initially prepared in the equilibrium ground state, the adiabatic cycle brings all bosons into the first excited one-particle state, leaving the system in a nonequilibrium state. The absorbed energy during the cycle is proportional to the number of bosons.
\end{abstract}

Keywords: Bose particles, adiabatic cycle, $\delta$-function potential, boson.

Received: 10 July 2016

Revised: 30 July 2016

\section{Introduction}

Since the discovery of the geometric phase [1], it has been widely recognized that adiabatic cyclic parameter variation does not necessarily bring a quantum eigenstate of a system back to itself. In general, an "exotic" quantum holonomy [2,3], in which one eigenstate turns into another eigenstate belonging to the same Hamiltonian, can be observed after the cyclic variation of the system parameters. In this paper, we examine an adiabatic cycle that excites a system consisting of Bose particles confined in a one-dimensional box. During the cycle, we vary an additional wall adiabatically, while the interparticle interaction is kept fixed. This is in contrast to the scheme described in $[4,5]$, where the interaction strength between Bose particles is an effective adiabatic parameter. In this study, we suppose that the wall is described by a $\delta$-function shaped potential [6-8]. We show that the first excited one-particle state is occupied by all the bosons to achieve the population inversion completely, if the system is prepared to be in the ground state. Namely, the energy gained by the bosons during the adiabatic cycle is proportional to the number of bosons.

The adiabatic cycle described above has a immediate relevance to the population inversion in Bose gas system [9], particularly in the super-Tonks-Girardeau gas, which has attracted considerable recent attention in both experimental and theoretical studies [4,10-12]. In the super-Tonks-Girardeau gas, which may be described by the Lieb-Liniger model [13] with strongly attractive interaction, the population inversion is created through an "adiabatic" process, where the interaction strength is suddenly flipped from infinitely repulsive to infinitely attractive [4, 14]. Such a population inversion can be induced even by an adiabatic cycle, which can be obtained with an extension of the adiabatic process that connects Tonks-Girardeau and super-Tonks-Girardeau gases both to weaker repulsive and weaker attractive regime. The repetitions of this adiabatic cycle transform the ground state of non-interacting bosons into their higher excited states and achieve the population inversion [5]. This is counterintuitive, since there is no external field to drive the final state of the bosons away from the initial state.

The paper is organized as follows: in Section 2, the basic setup of our model is presented in the simplest setting of one boson; Section 3 and 4 treat several interacting and non-interacting bosons, respectively; Section 5 contains the summary and the discussion. .

\section{A particle in a box with a $\delta$-wall}

In order to examine $N$ Bose particles in a one-dimensional box with an additional $\delta$-wall, we review the single particle case, i.e., $N=1$ [8], where the system is described by the Hamiltonian:

$$
H(g, X)=\frac{p^{2}}{2 m}+V(x)+g \delta(x-X),
$$

where $m$ is the particle mass, $V(x)$ is the confinement potential, and $g$ and $X$ are the strength and position of $\delta$-wall. In particular, we assume that $V(x)$ describes an infinite square well with the length $L$, i.e., $V(x)=0$ for $0<x<L$ and $V(x)=\infty$ otherwise [6,7].

We introduce an adiabatic cycle $C$, which consists of three adiabatic processes $C_{I}, C_{I I}$ and $C_{I I I}$, as shown in Fig. 1. We suppose that the $\delta$-wall is initially absent, i.e., $g=0$ in 1 , and that the system is in a stationary state initially. In the first part of $C$, which will be called as $C_{I}$, an impenetrable wall is inserted at $x_{0}$ adiabatically. In 
terms of the $\delta$-wall, the strength $g$ is slowly increased from 0 to $\infty$, while its position $X$ is fixed at $x_{0}$ during $C_{I}$. Subsequently, in the second part $C_{I I}$, the position $X$ of the impenetrable wall is adiabatically changed from $x_{0}$ to $x_{1}$. In the last part $C_{I I I}$, the $\delta$-wall at $X=x_{1}$ is adiabatically turned off. At the end of the cycle $C$, the $\delta$-wall has no effect, again.

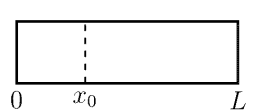

(a)

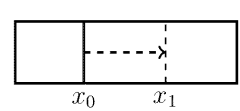

(b)



(c)

FIG. 1. The adiabatic cycle $C$ of a one-dimensional box, which contains Bose particles. The strength and the position of an additional $\delta$-wall is adiabatically varied during $C$

In Fig. 2, we depict the parametric dependence of eigenenergies of the single-particle Hamiltonian $H(g, X)(1)$ along $C$. Throughout this manuscript, we indicate the eigenenergy $E$ using a normalized wavenumber $\bar{k}$ :

$$
\bar{k} \equiv \sqrt{\frac{E}{N \epsilon}},
$$

where $\epsilon=(\hbar \pi / L)^{2} /(2 m)$ is the ground eigenenergy of the particle in the infinite square well.

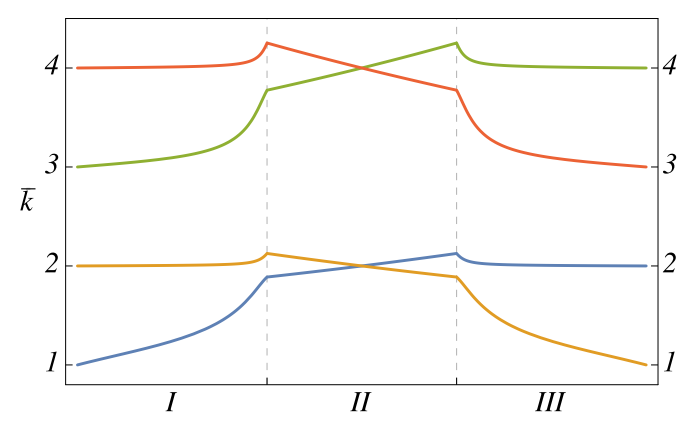

FIG. 2. Parametric evolution of eigenenergies with $N=1$ along the cycle $C$, which consists of $C_{I}$ (left part), $C_{I I}$ (middle part) and $C_{I I I}$ (right part). The eigenenergies are depicted by their normalized wavenumber $\bar{k}(2)$. We set $x_{0}=0.4703 L$ and $x_{1}=L-x_{0}$

The adiabatic time evolution of the single-particle system along $C$ depends on $x_{0}$ and $x_{1}$. In the following, we explain the case $\frac{2}{5} L<x_{0}<\frac{1}{2} L<x_{1}<\frac{3}{5} L$, which may be explained from Fig. 2. A more rigorous argument is found in [8].

First, let us consider the case that the initial state is the ground state $\left|1\left(g=0, X=x_{0}\right)\right\rangle$ of the particle in the infinite square well, where $|n(g, X)\rangle$ denotes the $n$-th adiabatic eigenstate of $H(g, X)$ during processes $C_{I}$ and $C_{I I I}$. We will omit to indicate $(g, X)$ in the following. After the completion of $C_{I}$, the state vector arrives at $\left|R_{1}\right\rangle$, the ground state of the right well, since we choose the right well in $C_{I I}$ is slightly larger than the left well. During $C_{I I}$, there occurs a spectral degeneracy between $\left|R_{1}\right\rangle$ and $\left|L_{1}\right\rangle$, the ground state of the left well. This is because the size of the left (right) well is increasing (decreasing) during $C_{I I}$, and these sizes coincide at $X=L / 2$. At the end of $C_{I I},\left|R_{1}\right\rangle$ becomes the first excited state, which adiabatically continued to $|2\rangle$, which is the second excited state of the particle in an infinite square well, through $C_{I I I}$. Hence, the "population inversion" in the single-particle system occurs if the system is prepared to be in the ground state initially.

Second, we examine the case that the initial state is the first excited state $|2\rangle$, which offers the "inverse" of the population inversion. Through the adiabatic cycle $C$, the system arrives at $\left|L_{1}\right\rangle$ after the completion of $C_{1}$, and then arrives at $|1\rangle$ at the end of the cycle $C$. Namely, either $|1\rangle$ and $|2\rangle$ return to the initial states after the completion of the adiabatic cycle $C$ twice.

Third, let us examine the cases that the initial states are $|3\rangle$ and $|4\rangle$, which are the first and second excited state, respectively. Now, $C$ induces an interchange of these two states, through the intermediate states $\left|R_{2}\right\rangle$ and $\left|L_{2}\right\rangle$, which are localized the right and left well during the process II.

A similar interchange of initial eigenstates occurs as a result of the adiabatic cycle $C$, as long as we choose $x_{0}$ and $x_{1}$ appropriately. In general, the level crossing of the one-particle Hamiltonian (1) during the process $C_{I I}$ 
plays an important role to determine which pairs of eigenstates are interchanged by $C$, while there is no level crossing generically during the processes $C_{I}$ and $C_{I I I}[8]$.

We will now comment on the stability of the present scheme for the one-body population inversion. A crucial point is the stability of the adiabatic time evolution across the level crossing during $C_{I I}$. The level crossing may be lifted due to an imperfection of the impenetrable wall, i.e., the $\delta$-wall with an infinite strength. If the level splitting is small enough, we may employ the diabatic process around the avoided crossing to realize the one-body population inversion. It has been shown that an open diabatic process made of a time-dependent potential well produces a second excited state from the ground state of a Bose particle [17]. This diabatic scheme is applied to create collective excitations of interacting bosons [17-19].

\section{Non-interacting bosons}

We first examine the case that the number of the Bose particles is $N$, assuming the absence of interparticle interaction. It is straightforward to extend the above result for $N=1$, once we restrict the case that $N$ bosons initially occupies the one-particle state $|n\rangle$. Hence, the system is in an adiabatic state of the $N$ Bosons:

$$
\left|n^{\otimes N}\right\rangle \equiv|n n . . n\rangle
$$

where the one-particle adiabatic state $|n\rangle$ is occupied by $N$ bosons, during $C_{I}$ and $C_{I I I}$.

If there is no interparticle interactions, the parametric evolution of averaged wavenumber $\bar{k}$ for the adiabatic $N$-particle state agrees with the one for the single-particle system. This suggests that the adiabatic cycle $C$ of the $N$-particle system with no interaction delivers the ground state $\left|1^{\otimes N}\right\rangle$ to the excited state $\left|2^{\otimes N}\right\rangle$, i.e., the complete population inversion, as is seen in Figure 2. The energy that the particles acquire during the cycle $C$ is proportional to the number of the particles.

\section{Interacting bosons}

We now examine the adiabatic cycle $C$ for $N$ interacting Bose particles. We mainly examine the case that the system is initially in the ground state. In order to confirm that the $N$-particle population inversion really occurs, we need to examine the effect of the interparticle interaction.

We assume that the interparticle interaction $V$ consists of two-body contact interactions. Namely, we suppose that $V$ takes the following form:

$$
V\left(x_{1}, x_{2}, \ldots, x_{N}\right)=\lambda \sum_{\langle i, j\rangle} \delta\left(x_{i}-x_{j}\right),
$$

where $\lambda$ is the interaction strength, and the summation is taken over pairs. We also assume that the interparticle interaction is weak enough so that the topology of the parametric dependence of eigenenergy remain unchanged, except around the level crossing points of the noninteracting bosons. Namely, when the gaps of the eigenenergies between neighboring levels in the noninteracting system are larger than a constant value, the interparticle interaction shifts the eigenenergy at most $\mathcal{O}(\lambda)$, according to the standard perturbation theory. For small enough perturbative energy correction, the corresponding adiabatic time evolution of the stationary state of the interacting bosons closely follows the evolution of noninteracting bosons.

Accordingly, under the weak interparticle interaction condition, the eigenstates of the interacting bosons can be labeled by the quantum numbers of the noninteracting bosons. For example, the ground state of the initial and final points of the adiabatic cycle $C$ may be denoted as $\left|1^{\otimes N}(\lambda)\right\rangle$, whose overlapping integral with the unperturbed state $\left|1^{\otimes N}\right\rangle$ is large. Also, $\left|1^{\otimes N}(\lambda)\right\rangle$ can be constructed by the standard perturbation theory with a small parameter $\lambda$.

On the other hand, even a weak interparticle interaction can strongly influence the parametric evolution of energy levels in the vicinity of level crossings by making avoided crossings. Hence, we need to closely examine the level crossing of the non-interacting Bose particles.

In the following, we argue that the adiabatic time evolution closely follows that of the noninteracting system examined above, if the number of the particles is large enough. The key is the selection rule for the matrix element of $V$ in the adiabatic representation in the vicinity of the level crossings of non-interacting Bosons.

\section{1. "Tunneling" and direct contributions of the interaction in $N=2$}

We show that the effect of the interparticle interaction is significantly different, depending on whether a level crossing locates either in $C_{I I}$, or in $C_{I} \cup C_{I I I}$, as for the two body case. In the former case, the relevant matrix elements may be small since it involves only tunneling processes through the impenetrable wall. On the other hand, in processes $C_{I}$ and $C_{I I I}$, the matrix element cannot be negligible. However, it seems that there is no corresponding level crossing that affects the population inversion whose initial state is the ground state. 
The parametric evolutions of eigenenergies of the noninteracting two particle system are depicted in Fig. 3, in terms of the averaged wavenumber $\bar{k}$ (see, (2)). The parametric evolution of the eigenenergy that connects $|11\rangle$ and $|22\rangle$ has a level crossing with two eigenenergies during $C_{I I}$. The initial states of these energy levels are $|22\rangle$ and $|12\rangle$, which are $\left|L_{1} L_{1}\right\rangle$ and $\left|R_{1} L_{1}\right\rangle$ during $C_{I I}$, respectively.

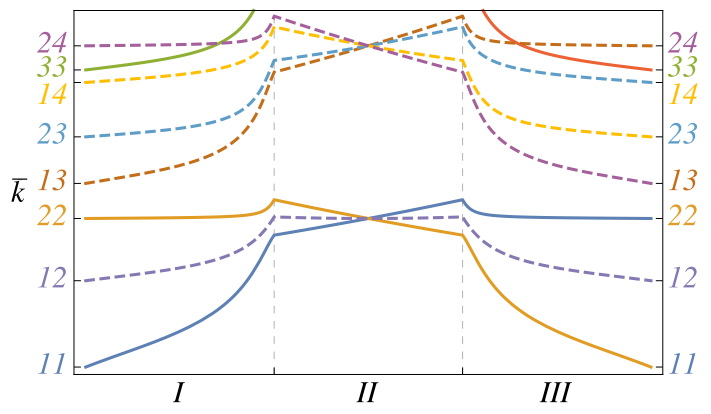

FIG. 3. Parametric evolution of normalized wavenumbers for $N=2$. Full lines indicate the levels whose initial states are $|n n\rangle(n=1, \ldots, 4)$ Other levels are depicted by dashed lines. Parameters are the same as in Fig. 2

We examine the matrix elements of the interparticle interaction term $V$ between the adiabatic basis vectors $\left|R_{1} R_{1}\right\rangle,\left|L_{1} L_{1}\right\rangle$ and $\left|R_{1} L_{1}\right\rangle$. Note that $\left|R_{1} R_{1}\right\rangle$ corresponds to the initial state $|11\rangle$ of the adiabatic cycle:

$$
\left\langle R_{1}, L_{1}|V| R_{1}, R_{1}\right\rangle=\sqrt{2} \lambda \int_{0}^{L}\left\{\psi_{R_{1}}(x) \psi_{L_{1}}(x)\right\}^{*}\left\{\psi_{R_{1}}(x)\right\}^{2} d x,
$$

for example. Since the single-particle adiabatic eigenfunctions $\psi_{L_{1}}(x)$ and $\psi_{R_{1}}(x)$ are completely localized in the left and right wells, respectively, the overlapping integral is zero, if the $\delta$-wall is completely impenetrable during $C_{I I}$. The level crossing accordingly remains even in the presence of the interparticle interaction. Thus the adiabatic cycle $C$ induces complete population inversion from $|11\rangle$ to $|22\rangle$, as in the non-interacting case.

Let us examine the case that the $\delta$-wall during $C_{I I}$ allows the tunneling leakage of particles due to some imperfections. Still, we may expect that the matrix elements due to the tunneling corrections are exponentially small. Since the resultant energy gap of the avoided crossing is also exponentially small, we may expect that the diabatic process easily almost recovers the complete population inversion.

Also, during the second process $C_{I I}$, the left and right part of the well may be separated. This allows us to make the tunneling correction arbitrarily small. Accordingly, the adiabatic limit that follows the extremely small avoided crossing would be difficult to realize.

Conversely, if the level crossing appears during $C_{I}$ or $C_{I I I}$, the interparticle interaction destroys the level crossing. In Fig. 3, such an example is seen between the levels whose initial states are $|33\rangle$ and $|24\rangle$, which is delivered to $\left|R_{2} R_{2}\right\rangle$ and $\left|L_{1} L_{2}\right\rangle$, respectively, in the absence of $V$.

The matrix element $\langle 33|V| 24\rangle$ does not vanish in general, since the relevant single-particle adiabatic eigenfunctions extend the whole box. Accordingly, the level crossings are destroyed to form avoided crossing. Thus the adiabatic process $C_{I}$ for example, delivers $|33\rangle$ and $|24\rangle$ at the initial point of $C_{I}$, to $\left|L_{1} L_{2}\right\rangle$ and $\left|R_{2} R_{2}\right\rangle$, respectively. This breaks the population inversion whose initial state is a higher excited state, e.g., the adiabatic cycle $C$ delivers $|33\rangle$ to $|44\rangle$ in the absence of interparticle interaction.

\subsection{Selection rule for $N=3$}

Here, we show that the interparticle interactions do not suppress the population inversion for $N>2$ due to a selection rule of $V$.

We explain this with the case $N=3$ (Fig. 4). Let us examine the level whose initial state is $\left|1^{\otimes 3}\right\rangle$ along $C$. The corresponding final state is $\left|2^{\otimes 3}\right\rangle$ in the absence of interparticle interaction.

First, the interparticle interaction has no, or exponentially small effect on the level crossing during $C_{I I}$, as shown in the case of $N=2$.

Second, we examine the level crossing in $C_{I I I}$, where the levels whose final states are $\left|2^{\otimes 3}\right\rangle$ and $|113\rangle$ exhibit crossing. We examine the matrix element of interparticle interaction $\left\langle 113|V| 2^{\otimes 3}\right\rangle$, which vanishes since $V$ is a two-body interaction, and the set of quantum numbers $(1,1,3)$ and $(2,2,2)$ has no common quantum number. 


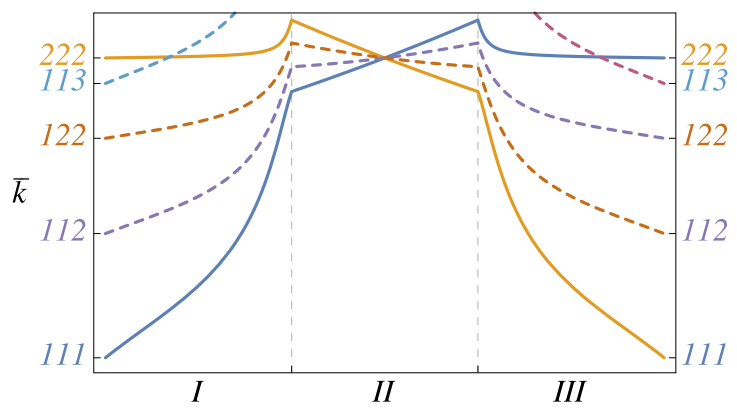

FIG. 4. Parametric evolution of normalized wavenumbers for $N=3$. Other parameters are the same as in Fig. 3

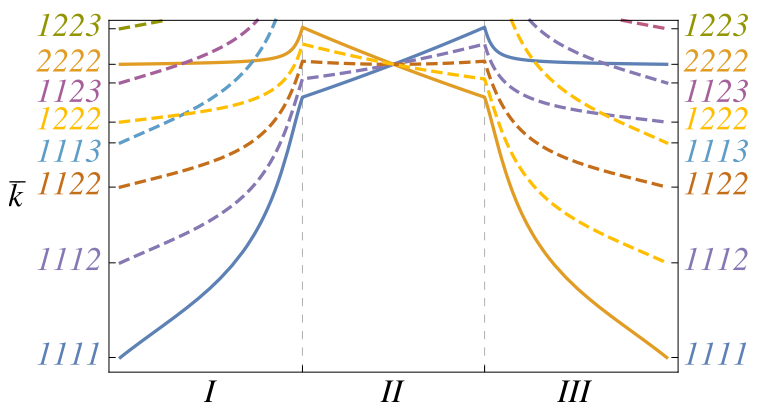

FIG. 5. Parametric evolution of normalized wavenumbers for $N=4$. Other parameters are the same as in Fig. 3

Still, there may be a tiny avoided crossing whose magnitude can be explained by the standard second-order perturbation theory. We may expect that the diabatic process induces complete population inversion whose final state is $\left|2^{\otimes 3}\right\rangle$. Also, even if the interaction strength $\lambda$ is moderately large, where the topology of the level diagram remains unchanged except that the avoided crossing becomes noticeable, the final state should be $|113\rangle$, whose energy is far larger than the ground state. In this sense, an incomplete population inversion should be realized.

\subsection{The population inversion for $N>2$}

We shall prove that the adiabatic cycle $C$ delivers $\left|1^{\otimes N}\right\rangle$ to $\left|2^{\otimes N}\right\rangle$ for $N>2$, even in the presence of two-body interparticle interaction. Here, we explain the selection rule for arbitrary $N(>2)$, and examine each part of the cycle $C$. For example, $N=4$ case is shown in Fig. 5.

We explain the selection rule of the two-body interparticle interaction for $N>2$. Namely, we examine the matrix element $\left\langle n_{1}^{\prime} n_{2}^{\prime} \ldots n_{N}^{\prime}|V| n_{1} n_{2} \ldots n_{N}\right\rangle$. The matrix element vanishes when the two sets of quantum numbers $\left(n_{1}^{\prime}, n_{2}^{\prime}, \ldots, n_{N}^{\prime}\right)$ and $\left(n_{1}, n_{2}, \ldots n_{N}\right)$ have at least three different elements, i.e., the number of the common quantum numbers is equal to $N-3$ or less. In other words, non-vanishing matrix element has the following $\left\langle n_{1}^{\prime} n_{2}^{\prime} n_{3} \ldots n_{N}|V| n_{1} n_{2} n_{3} \ldots n_{N}\right\rangle$ where $\left(n_{3}, \ldots, n_{N}\right)$ are the common quantum numbers.

We examine the first part $C_{I}$ of $C$. We assume that the system is initially in the ground state $\left|1^{\otimes N}(\lambda)\right\rangle$. According to the selection rule, it is sufficient to examine $\left|1^{\otimes N-2} \psi, \phi\right\rangle$, where $|\psi\rangle$ and $|\phi\rangle$ are single particle adiabatic states, e.g. $|2\rangle$. Now, we examine whether the eigenenergies of these states are degenerate. This is equivalent to comparing the eigenenergies corresponding to $|11\rangle$ and $|\psi, \phi\rangle$ of the two particle system. As is seen in Fig. 3, there is no level crossing in $C_{I}$. In this sense, there is no effective level crossing with the level $\left|1^{\otimes N}(\lambda)\right\rangle$, during $C_{I}$.

As for $C_{I I I}$, we conclude from a similar argument above, that the energy level corresponding to $\left|2^{\otimes N}(\lambda)\right\rangle$ has no effective level crossing.

Next, we examine $C_{I I}$, where the system is in $\left|R_{1}^{\otimes N}\right\rangle$. According to the selection rule, it suffices to examine $\left|R_{1}^{\otimes N-2} \psi, \phi\right\rangle$ with single particle adiabatic states $|\psi\rangle$ and $|\phi\rangle$. To clarify the level crossing, we compare $\left|R_{1} R_{1}\right\rangle$ with $|\psi, \phi\rangle$. There are three cases. First, the levels corresponding to $\left|R_{1} R_{1}\right\rangle$ and $\left|R_{n}, R_{n^{\prime}}\right\rangle\left(\left(n, n^{\prime}\right) \neq(1,1)\right)$ do not occur. Second, the levels corresponding to $\left|R_{1} R_{1}\right\rangle$ and $\left|R_{n}, L_{n^{\prime}}\right\rangle$ exhibit a degeneracy only when $n=1$ and $n^{\prime}=1$, where the corresponding matrix element involves a single-particle tunneling. Third, the levels corresponding to $\left|R_{1} R_{1}\right\rangle$ and $\left|L_{n}, L_{n^{\prime}}\right\rangle$ exhibit a degeneracy only when $n=1$ and $n^{\prime}=1$, where the corresponding matrix element involves two-particle tunneling. Since the matrix elements involving tunneling contribution are exponentially small, the resultant gap should also be small. Hence, the diabatic process should occur even when the speed of the impenetrable wall is moderately slow.

\section{Discussion and summary}

Here, we argue that the experimental realization of the population inversion suggested in this paper is feasible with the current state of the art. For example, we may utilize the scheme [20] to realize $\delta$-wall with an approximate Gaussian wall.

Another possibility is to use a heavy particle as a wall, whose position may be manipulated by, say, an optical tweezer. The effective interaction between the wall particle and other particle may be tuned by external fields. 
We note that the present scheme may offer a way to realize other exotic nonequilibrium states. Let us suppose, for example, the state of bosons is in $\left|2^{\otimes N}(\lambda)\right\rangle$, which can be generated from the adiabatic cycle $C$. After the interparticle interaction $\lambda$ is adiabatically increased to $\infty$, the system arrives the higher excited state of the TonksGirardeau system, which may be described by the Lieb-Linigher model with the infinite interparticle interaction strength [21,22]. Similarly, after $\lambda$ is adiabatically decreased to $-\infty$, the system now arrives at the higher excited state of the super-Tonks-Girardeau system [4]. This state is a much more highly-excited state compared to the super-Tonks-Girardeau state, because the initial state $\left|2^{\otimes N}(\lambda)\right\rangle$ is a higher excited state of noninteracting bosons.

We note that the present scheme offers a way to realize the super-Tonks-Girardeau gas in a one-dimensional box. For example, once we obtain $\left|2^{\otimes N}\right\rangle$ from $\left|1^{\otimes N}\right\rangle$ through the adiabatic cycle $C$, the system arrives at the super-Tonks-Girardeau gas as the strong attractive interparticle interaction is imposed. This scheme do not require neither the preparation of the Tonks-Girardeau gas [21,22] nor the sudden flip of the interparticle potential [4].

In summary, we have shown that the adiabatic cycle $C$ induces the nearly complete population inversion of the multi-boson system, when the interparticle interaction is not too strong. As pointed out in [8], for a single particle case, the present scheme may be extended to the case of an arbitrary shape of the confinement potential $V(x)$.

\section{Acknowledgements}

This research was supported by the Japan Ministry of Education, Culture, Sports, Science and Technology under the Grant number $15 \mathrm{~K} 05216$.

\section{References}

[1] Berry M.V. Quantal Phase Factors Accompanying Adiabatic Changes. Proc. Roy. Soc. A, 1984, 392, P. $45-57$.

[2] Tanaka A., Miyamoto M. Quasienergy Anholonomy and its Application to Adiabatic Quantum State Manipulation. Phys. Rev. Lett., 2007, 98, P. 160407.

[3] Tanaka A., Cheon T. Bloch Vector, Disclination and Exotic Quantum Holonomy. Phys. Lett. A, 2015, 379, P. 1693-1698.

[4] Haller E., Gustavsson M., et al. Realization of an Excited, Strongly Correlated Quantum Gas Phase. Science, 2009 , 325, P. $1224-1227$.

[5] Yonezawa N., Tanaka A., Cheon T. Quantum Holonomy in the Lieb-Liniger Model. Phys. Rev. A, 2013, 87, P. 062113.

[6] Flügge S. Practical Quantum Mechanics, vol. 1. Berlin, Springer-Verlag, 1971.

[7] Ushveridze A.G. Analytic Properties of Energy Levels in Models with Delta-function Potentials. J. Phys. A, 1988, 21, P. 955-970.

[8] Kasumie S., Miyamoto M., Tanaka A. Adiabatic Excitation of a Confined Particle in One Dimension with a Variable Infinitely Sharp Wall. Phys. Rev. A, 2016, 93, P. 042105.

[9] Grynberg G., Aspect A., Fabre C. Introduction to Quantum Optics. Cambridge, Cambridge University Press, 2010.

[10] Astrakharchik G.E., Boronat J., Casulleras J., Giorgini S. Beyond the Tonks-Girardeau Gas: Strongly Correlated Regime in Quasi-OneDimensional Bose Gases. Phys. Rev. Lett., 2005, 95, P. 190407.

[11] Olshanii M. Atomic Scattering in the Presence of an External Confinement and a Gas of Impenetrable Bosons. Phys. Rev. Lett., 1998, 81, P. 938-941.

[12] Guan X. Critical Phenomena in One Dimension from a Bethe Ansatz Perspective. Int. J. Mod. Phys. B, 2014, 28, P. 1430015.

[13] Lieb E.H., Liniger W. Exact Analysis of an Interacting Bose Gas. I. The General Solution and the Ground State. Phys. Rev., 1963, 130, P. $1605-1616$.

[14] Girardeau M. Relationship between Systems of Impenetrable Bosons and Fermions in One Dimension. J. Math. Phys., 1960, 1, P. 516-523.

[15] Vitanov N.V., Halfmann T., Shore B.W., Bergmann K. Laser-induced Population Transfer by Adiabatic Passage Techniques. Annu. Rev. Phys. Chem., 2001, 52, P. 763-809.

[16] Guérin S., Yatsenko L.P., Jauslin H.R. Dynamical Resonances and the Topology of the Multiphoton Adiabatic Passage. Phys. Rev. A, 2001, 63, P. 031403(R).

[17] Karkuszewski Z.P., Sacha K., Zakrzewski J. Method for Collective Excitation of a Bose-Einstein Condensate. Phys. Rev. A, 2001, 63, P. 061601.

[18] Damski B., Karkuszewski Z.P., Sacha K., Zakrzewski J. Simple Method for Excitation of a Bose-Einstein Condensate. Phys. Rev. A, 2002, 65, P. 013604.

[19] Karkuszewski Z.P., Sacha K., Smerzi A. Mean Field Loops versus Quantum Anti-crossing Nets in Trapped Bose-Einstein Condensates. Eur. Phys. J. D, 2002, 21, P. 251-254.

[20] Meyrath T.P., Schreck F., et al. Bose-Einstein Condensate in a Box. Phys. Rev. A, 2005, 71, P. 041604(R).

[21] Paredes B., Widera A., et al. Tonks-Girardeau Gas of Ultracold Atoms in an Optical Lattice. Nature, 2004, 499, P. $277-281$.

[22] Kinoshita T., Wenger T., Weiss D.S. Observation of a One-Dimensional Tonks-Girardeau Gas. Science, 2004, 305, P. 1125-1128. 